\title{
Role of Noncoding RNAs in Chronic Pancreatitis
}

\author{
BISHNUPRIYA CHHATRIYA ${ }^{1}$, PIYALI SARKAR ${ }^{1}$ and SRIKANTA GOSWAMI ${ }^{1, *}$ \\ ${ }^{I}$ National Institute of Biomedical Genomics, Kalyani, West Bengal, India
}

(Received on 29 November 2017; Revised on 02 February 2018; Accepted on 30 March 2018)

\begin{abstract}
Chronic pancreatitis (CP) is a progressive inflammatory disease of pancreas, resulting in irreversible damage of exocrine and endocrine function of the organ. Tissue fibrosis is a characteristic feature of $\mathrm{CP}$, where the pancreatic stellate cells have been thought to play a very crucial role. Patients with chronic pancreatitis have also been reported to be more susceptible to pancreatic cancer as compared to any healthy individual. Considering the public health importance of the disease, efforts are underway to comprehend the alterations in gene expression pattern during the development and progression of the disease. Non-coding RNAs (ncRNAs) do not code for any proteins, but they are the key players in modulating the expression of large number of protein coding genes, consequently maintaining their normal function as well as adversely affecting the gene expression leading to disease pathophysiology. In recent years, studies on ncRNAs have gained much importance with the discovery of more and more types of ncRNAs and their involvement in almost every disease. Same is true for chronic pancreatitis as well, and the ncRNAs have been implicated to have important regulatory role in the biology of this disease. Furthermore, they have also been shown to have the ability to be used as diagnostic and prognostic biomarkers of this disease. This review highlights the major findings in the field of ncRNAs (especially microRNAs and long noncoding RNAs) with respect to chronic pancreatitis in humans.
\end{abstract}

Keywords: Noncoding RNA; Chronic Pancreatitis; disease

\section{Introduction}

Chronic inflammatory diseases have always been on the radar due to high chances of their development into cancer. Chronic pancreatitis is of no exception and both clinical and experimental studies have shown strong association to pancreatic cancer development. Apart from that, because of its irreversible nature, the disease is often associated with high degree of morbidity and mortality with a huge impact on the public health burden. Therefore, there has been a long lasting interest among the scientific community to understand the development and progression of this disease, especially at the molecular level.In recent years, research on noncoding RNAs (ncRNAs) has gained much importance with the discovery of newer members through technologically advanced methods, as well as with more studies establishing their functional relevance in the disease pathophysiology. Furthermore, in addition to their regulatory role, the noncoding RNAs have also been implicated as potential candidates for diagnostic or prognostic biomarkers of various diseases, following their detection in plasma/serum and other body fluids and with their presence as one of the major contents of exosomes and microvesicles. As a consequence, following the global trends, there has been a lot of studies to investigate the contribution of noncoding RNAs in the disease biology of chronic pancreatitis and also to find out whether the alteration in noncoding RNA pattern in peripheral blood or other body fluids could function as early diagnostic or prognostic biomarker of the disease. Here, we aim to review the important findings in order to revisit our achievement in this field and also to explore how we can move forward with our existing knowledge.

\section{Describing the Disease: Chronic Pancreatitis}

'Chronic Pancreatitis', as the name indicates, is chronic inflammation of pancreas. It is characterized by progressive and irreversible damage to both 
exocrine and endocrine components of the pancreas, therefore, leading to exocrine insufficiency and diabetes mellitus. Symptoms of the disease include characteristic pain in abdomen radiating towards the back, steatorrhoea, fibrosis and atrophy of pancreas accompanied by ductal irregularities. It is a multifactorial disorder with alcohol being one of the major factors responsible for the development of chronic pancreatitis (also known as alcoholic chronic pancreatitis or ACP) worldwide (Clemens et al., 2010). Apart from this, hereditary chronic pancreatitis (HCP) is a very rare form of early onset chronic pancreatitis with a classic autosomal dominant nature of inheritance, involving mutations in specific genes (Rosendahl et al., 2007; Kim et al., 2004). However, chronic pancreatitis may also occur without an apparent cause, in which case the term "idiopathic chronic pancreatitis" (ICP) is used (Dodge, 1998). The scenario in a tropical country like India is quite different. Majority of the cases here are not attributed to alcohol and prevalence of ICP is more. Though a predominant subtype of ICP with early age at onset and severe ductal calcifications had earlier been identified as 'tropical calcific pancreatitis' or 'tropical chronic pancreatitis'(TCP) by some Indian groups (Barman et al., 2003); further studies suggest that the phenotype of the disease has changed and the term 'tropical pancreatitis' could be considered misnomer (Midha et al., Gut 2010). Genetic factors have reportedly been found to be associated with the disease with various studies identifying mutations or polymorphisms in genes like protease, serine-1 (PRSS1), protease, serine-2 (PRSS2), serine peptidase inhibitor Kazal type-1 (SPINK1), cystic fibrosis transmembrane conductance regulator (CFTR), chymotrypsin C (CTRC), calcium sensing receptor $(C A S R)$, cathepsin B (CTSB) and claudin 2 $(C L D N 2)$, modulating disease susceptibility in different forms of pancreatitis including chronic pancreatitis (Reddy et al., 2014; Mounzer et al., 2013). Overall, chronic pancreatitis is one of the most common sources of morbidity in the United States and Europe with annual incidence rates reported worldwide ranging from 5 to 14 per 100,000 individuals, with a prevalence of approximately $30-50$ per 100,000 individuals (Kleeff et al., 2017). Not many reports are there presenting the epidemiology of the disease in India. One study demonstrates the prevalence of the disease as high as $114-200$ cases per 100,000 populations in Southern India as per a recent survey conducted in different countries in the Asia-Pacific region (Garg et al., 2004). Over the years many theories for the pathogenesis of the disease have been proposed like oxidative stress theory, toxic metabolic theory, stone and ductal obstruction theory, necrosisfibrosis theory etc. (Stevens et al., 2004). However, Whitcomb and colleagues have proposed an interesting hypothesis for the pathogenic development of chronic pancreatitis, which unifies all these previous theories and also incorporates recent advancements about the cellular and molecular changes leading to the disease development. They have termed their hypothesis as 'Sentinel Acute Pancreatitis Event (SAPE) Hypothesis' (Schneide et al., 2002). This model describes factors associated with chronic pancreatitis fitting to a hypothetical pathway that leads from an episode of acute pancreatitis towards chronic pancreatitis. According to this model, an initiating event (sentinel event) is necessary for causing acute pancreatitis and acinar cell injury first, which is followed by recruitment and activation of the immune cells and stellate cells crucial for the development of chronic inflammation and fibrosis. This finding also highlights the importance of pancreatic stellate cells in the process. Pancreatic stellate cells (PSCs) have been found to play a very crucial role in the development of fibrosis. These are myo-fibroblast like cells present in pancreas in quiescent form. They get activated in the presence of inflammatory cytokines whenever there is any pancreatic injury. Upon activation, they migrate to the site of tissue injury and release extra-cellular matrix components to promote tissue repair. This is the normal function of PSCs but whenever there is chronic inflammation, this system goes aberrant leading to over activation of PSCs and causing tissue fibrosis (Stevens et al., 2004; Apte et al., 2015).

Nature of this disease is progressive and irreversible resulting in irreparable damage to the pancreas. Hence, there is high degree of morbidity and mortality associated with the disease. Management of the disease starts with lifestyle modifications like stopping alcohol and tobacco use and incorporating relevant dietary changes. Analgesics, anti-diabetics and pancreatic enzyme supplementation are commonly prescribed medicines. If there is requirement of endoscopic or surgical interventions, the risks and benefits of each procedure 
is critically evaluated. While therapeutic endoscopy is adopted for symptomatic or complicated pseudocyst, biliary obstruction, and decompression of pancreatic duct; surgical procedures are followed mainly for pancreatic head resection during the treatment of severe chronic pancreatitis as the pancreatic head mass lies at the core of the chronic inflammatory process. Over time, organ-preserving procedures, such as the duodenum-preserving pancreatic head resection and the pylorus-preserving Whipple, have become the standard treatment procedure for the surgeons and have resulted in major improvements in pain relief, salvation of pancreatic function, and therefore increasing the quality of life of patients (Strobel et al., 2009; Forsmark, 2013). However, there is no such drug which could be used to either slow down this debilitating disease or to reverse the pancreas to normalcy by targeting relevant signaling pathways. Hence, there should be more studies aiming to elucidate the molecular changes taking place during disease development and the progression that might be helpful in designing future strategies for diagnostic and therapeutic interventions for the treatment of pancreatitis.

\section{Overview of Noncoding RNAs}

Almost $75 \%$ of human genome is transcribed into RNA but only $2 \%$ of these transcripts code for proteins, which implies that most of the RNA diversity in our cells is due to non-coding RNAs (Alexandar et al., 2015). Non-coding RNAs themselves do not code for any proteins but instead regulate the expression of target genes. Non-coding RNAs could be classified as microRNAs, PIWI interacting RNAs (piRNAs), long noncoding RNAs (lncRNA), small nucleolar RNAS (snoRNAs), long intergenic noncoding RNAs (lincRNAs) etc. Among them, microRNAs (miRNAs) have been most well characterized. miRNAs are small ncRNAs of about 22 nucleotide length which are found to regulate around $60 \%$ or more protein coding genes (Esteller, 2011). They are capable of downregulating the expression of their target genes either by mRNA degradation or by inhibition of translation initiation. piRNAs are slightly longer than miRNAs with the length of around 24-30 nucleotide. They are thought to regulate gene expression together with the help of PIWI proteins. lncRNAs encompasses all the ncRNAs which are more than 200 nucleotide long. They are known to mediate epigenetic modifications of DNA by recruiting chromatin remodeling complexes to specific locus. lincRNAs are the class of lncRNAs transcribed from long intergenic regions. They regulate the expression of neighbouring genes, as well as distant sequences. Circular RNAs are very recent discoveries in the group of noncoding RNAs which belong to a large class of endogeneous RNAs believed to be originated by exon skipping events (Huang et al., 2017).

\section{Noncoding RNAs in Diseases}

Role of non-coding RNAs in disease is not new. Many of them are reported to be differentially expressed and play important role/s in development and pathophysiology of various diseases. Discussing all of them is beyond the scope for this review; however their role in cancer and inflammatory diseases is briefly discussed.

miRNAs have been explored thoroughly for their role in cancer. They act as oncomiR as well as tumor suppressor miRs and have key role in tumorigenesis process (Croce, 2009). miRNA deregulation could occur through epigenetic changes and genetic alterations which could cause problems associated with miRNA synthesis, maturation or binding to their targets (Esteller, 2011). piRNAs and its associated proteins are also involved in cancer development. PIWI associated RNAs are also found to be aberrantly expressed in human somatic tumors (Cheng, 2011). Among the lncRNAs, HOX transcript antisense RNA (HOTAIR), HOXA distal transcript antisense RNA (HOTTIP), Metastasis associated lung adenocarcinoma transcript 1 (Non-Protein Coding) (MALAT1), Hepatocellular carcinoma up-regulated long non-coding RNA (HULC) and H19 are very well studied in human neoplasia and with the advent next generation technologies new and new lncRNAs are being implicated in cancer (Bhan et al., 2017). Furthermore, circular RNAs are also being thought to play important role in oncogenesis through their regulation of transcription, RNA splicing and also through their function as microRNA sponge (Huang et al., 2017).

Non-coding RNAs has been found to play an important role in pathogenesis of various inflammatory diseases like obesity, atherosclerosis, type 2 diabetes, osteoarthritis, autoimmune and degenerative diseases, asthma, periodontitis, cirrhosis, as well as, Chronic 
Pancreatitis. Inflammation is mediated by various inflammatory mediators like Tumor Necrosis Factoralpha (TNF-alpha), Interleukin 1 (IL-1), Interleukin 6 (IL-6), Interleukin 18 (IL-18), Nuclear Factor Kappa B Subunit (NFkB), Intercellular Adhesion Molecule1 (ICAM1), Vascular Cell Adhesion Molecule-1 (VCAM1), Plasminogen Activator Inhibitor-1 (PAI1) etc. (Marques-Rocha et al., 2015). These mediators are activated by signals from the cell membrane in response to foreign antigens. They act as transcription factors to induce or repress certain genes involved in the inflammatory process. The noncoding RNAs play a role here by modulating the expression of inflammatory genes directly or by modulating the mediators, which in-turn affects the inflammatory genes (Carpenter et al., 2015). Furthermore, one of the most important discoveries in recent years is the detection of nuclease resistant extracellular miRNAs in almost all known biological fluids. Strong evidence suggests that some of these extracellular miRNA species could perform cell signaling function during disease development and progression. Their expressions have also been attributed to specific diseases. Therefore, a lot of work is being pursued to unfold the potential of these exosomal miRNAs as non-invasive biomarkers for various diseases.

\section{Role of miRNAs in Chronic Pancreatitis}

Inflammation is a protective mechanism through which further injury to the tissue is prevented by infiltration of granulocytes from the blood aiming to remove the cause of injury. Once the tissue damaging agent is eliminated, the process called resolution of inflammation starts, the purpose of which is to promote tissue repair and bring it back to homeostasis through inhibition of pro-inflammatory signaling, prevention of further granulocyte recruitment and clearance of the damaged tissue. However, during chronic inflammation, this resolution step is disrupted resulting in continuing inflammatory response and progressive damage of the tissue. Furthermore, chronic inflammatory conditions have also been very strongly linked to the development of malignancy. Rudolf Virchov, back in 1863, first time proposed the role of inflammation in carcinogenesis and since then multiple studies have established that chronic inflammation in esophagous, stomach, liver, pancreas, colon, thyroid and other organs can induce malignancy through generation of reactive oxygen and nitrogen species (ROS and RNS) and induction of genomic instability through several genetic and epigenetic changes (Balkwill et al., 2001; Ptak-Belowska et al., 2012). Chronic pancreatitis is no exception and several studies have reported the disease to be a risk factor for pancreatic ductal adenocarcinoma (Zheng et al., 2013).

miRNAs have already been described to play a significant role in the modulation of chronic inflammatory pathways through regulation of the expression of several pro and anti-inflammatory genes and few studies have also been done to evaluate their role in development and progression of chronic pancreatitis. Genome-wide comparison of miRNA expression between pancreatic tissue from $\mathrm{CP}$ patients and normal pancreatic tissue was reported in several studies. While the first one made comparison of miRNA profile of both blood and tissue from normal, CP and PDAC patients (Bauer et al., 2012); the second study used the same dataset and identified 14 upregulated and 39 downregulated miRNAs in pancreatitis samples compared with control samples, through a meta-analysis (Wang et al., 2016). They further identified 424 differentially expressed miRNA gene pairs in the miRecords and miRWalk databases and they got 290 target genes of differentially expressed miRNAs. The functions of target genes were predicted using the DAVID online tool and GO and KEGG pathway enrichment analyses was followed revealing the major biological pathways they contribute to. Some of the target genes like Cyclin D1 (CCND1), AKT Serine/Threonine Kinase 2 (AKT2), Cyclin Dependent Kinase 6 (CDKO) and SMAD Family Member 2 (SMAD2) were also involved in the pancreatic cancer pathway. They further performed regulatory network analysis and reported that 279 of these genes were pancreatitis associated genes, which were targeted by 37 miRNAs in the regulatory network. Another original study similarly compared miRNA expression profile from pancreatic tissues of normal, $\mathrm{CP}$ and PDAC patients to identify key changes in them. A set of 22 miRNAs showed increased expression in chronic pancreatitis and expression of 2 miRNAs were decreased compared with normal pancreas (Bloomston et al., 2007). The same dataset was also reanalyzed by another group and the results were integrated with gene expression data from pancreatic tissue in $\mathrm{CP}$ 
patients and healthy individuals (Wang et al., 2017). Initially, they identified 624 DEGs and 305 DEmiRs followed by clustering analysis, further identifying DEGs and DEmiRs differentially expressed between $\mathrm{CP}$ and normal samples. Gene set enrichment analysis resulted in 73 downregulated and 84 upregulated pathways; like extracellular matrix (ECM) receptor interaction, focal adhesion, cell adhesion molecules, vasculature development, cell migration and so on. Next, they performed the target analysis using DEG and DEmiRs and functional analysis of these differentially expressed targets were also done and DEmiR-risk gene-pathway relationships were also established. They further explored whether the miRNA expression profile of rat CP model is similar to their analysis of human $\mathrm{CP}$ patients and identified miR-182, miR-184, miR-205, and miR-431 upregulated in both human and rat $\mathrm{CP}$ pancreas; modulating pathways and processes related to regulation of apoptosis, regulation of phosphate metabolic process, neurotrophin signaling pathway and pancreatic cancer etc. Another important study also made similar comparison of miRNA expression in normal pancreas and pancreatic tissue from CP and PDAC patients (Szafranska et al., 2007). They have also included pancreatic cancer cell lines in the comparison increasing further interest. Their result showed that miRNA expression profiles in chronic pancreatitis were distinct from the normal and cancer profiles and interestingly, in most of the cases global miRNA expression levels in pancreatitis tissues were intermediate between the normal and PDAC tissues. Amongst the 68 miRNAs differentially expressed in chronic pancreatitis vs normal tissues, 61 were also deregulated in pancreatic cancer. However, they performed the target identification and function prediction analysis for only the top 26 miRNAs deregulated in PDAC. The list of top ten deregulated miRNAs identified in these studies is shown in Table 1. Moreover, identification of pancreatitis associated genes as targets of deregulated miRNAs and their involvement in relevant pathways leading to pancreatitis is further supported by studies which have independently identified deregulated genes as important factors in epithelial-stromal signaling in pancreatic desmoplasia (Binkley et al., 2004) and pancreatitis associated pancreatic fibrosis (Bai et al., 2016). In addition to these studies, there are few other studies where different groups have compared the expression of individual miRNAs to evaluate their functional role. Most of these studies have focused on PDAC and one of them has looked into the CP patients too where miR-217 has been reported to be downregulated in CP pancreas (VychytilovaFaltejskova et al., 2015). Reports from another group have also suggested a possible role for downregulated miR-217 (Deng et al., 2014). According to their study, miR-217 downregulation is involved in epithelialmesenchymal transition via miR-217-Sirtuin-1 (SIRT1) pathway.

It is very well known that alcohol is one of the major cause leading to CP. miR-34a has been found associated with alcohol related fibrosis in liver (Meng et al., 2012) and there is enough evidence that it is also differentially expressed in pancreas of CP patients (Hu et al., 2015). miR-34a modulates of expression of matrix metalloproteases 1 and 2 (MMP1, MMP-2), which are the mediators involved in cell remodeling during alcohol-induced fibrosis (Hu et al., 2015; Derdak et al., 2013). Increased expression of miR-21, miR-199a-3p and miR-211 and decreased expression of miR-148a and miR-802 in the pancreas of mice models upon ethanol feeding has also been reported (Charrier et al., 2014). Other groups have also suggested role of miR-21 in chronic pancreatitis and reported that a miR-21 inhibitor can prevent alcoholic pancreatitis and pancreatic fibrosis ( $\mathrm{Hu}$ et al., 2015; Natarajan et al., 2015). One important aspect is the involvement of pancreatic stellate cells (PSCs), which has been known to play an important role in fibrosis of pancreatic tissue. It has been mentioned before that they get activated by inflammatory cytokines. miRNAs plays an important role here as well. They modulate the expression of the inflammatory cytokines and thereby regulate the activation of stellate cells. Exploration of the role of miRNAs in PSC biology has not been started much before and there is almost no study investigating the changes in miRNA expression profile in human PSCs, linking them with PSC physiology. Very few studies have attempted in animal models and the most important onehave reported 42 upregulated and 42 downregulated miRNAs in activated pancreatic stellate cells as compared to thequiescent one in rat models (Masamune et al., 2014). Pathway analysis of target genes revealed signaling pathways such as p38 MAP kinase, extracellular signal regulated kinases, and Smad2/3. Insulin and proinflammatory cytokine 
signaling pathways were also reported which indicates the role of miRNAs in modulation of inflammatory proteins. The same group later on identified 251 miRNAs enriched in PSC derived exosomes in human subjects. Highly expressed microRNAs included miR1246, miR-21-5p, and miR-1290, which are also reported to be found in serum of pancreatic cancer patients (Takikawa et al., 2017). So, clearly these miRNA alterations along with gene expression result in PSCs could explain the biology of these cells towards pathophysiology of CP and PDAC and could be used as biomarkers in pancreatic cancer as well.

Chronic pancreatitis is a disease which is diagnosed by its typical symptoms and imaging findings of atrophic pancreas or pancreatic calcification through CT-scan, MRCP, ERCP etc. But, unfortunately, when these symptoms are manifested in a patient, the disease has already been progressed to late stage causing irreversible damage to the organ. There are no early diagnostic non-invasive biomarkers for the disease and recent efforts have also explored the possibility of finding blood based miRNA biomarkers for early detection of chronic pancreatitis. One such studyhas reported six serum miRNAs which have the potential to be used as the biomarker for diagnosis and prognosis of CP (Xin et al., 2017). According to their study, miR-130a and miR-221 could distinguish early $\mathrm{CP}$ from late $\mathrm{CP}$ whereas miR-320a, miR-320b, miR-320c and miR-320d are specific for late CP (Table 1). Together, these six miRNAs could distinguish $\mathrm{CP}$ from other similar health conditions. However, more similar studies need to be conducted to establish them as a diagnostic or prognostic biomarker at the clinical level. Additionally, there are a few studies where alterations in specific miRNAs have been looked into serum or plasma of CP patients. miR-155, miR-196a, miR-181a, miR-181b are reported to be overexpressed in plasma of $\mathrm{CP}$ patients as compared to the normal healthy controls (Liu et al., 2012). miR-21 is found to be upregulated in serum of $\mathrm{CP}$ patients as reported by another group (Kong et al., 2011). In addition to serum or plasma based detection of extracellular miRNA, there are reports of investigating urinary or salivary miRNAs also for their biomarker potential. Following the same tune, one group has conducted a study and identified 4 upregulated and 11 downregulated miRNAs in urine of CP patients as compared to the healthy individuals (Debernardi et al., 2015). Being the first of its kind of report, the study has important implications in terms of future diagnostic potential of urinary miRNAs.

\section{Role of Long Noncoding RNAs in Chronic Pancreatitis}

Advancements of genome sequencing technologies have helped us in identifying large numbers of long (> $200 \mathrm{nt}$ ) noncoding RNAs (lncRNAs) mapping to either intronic or intergenic regions. Interestingly, some of them are poly-adenylated and some of them are non-adenylated too. There is also a huge amount of interest to decode the functional role of this subset of noncoding RNAs and coordinated efforts throughout the globe has assigned their roles in important biological processes. The role of H19 and X Inactive Specific Transcript (XIST) long noncoding RNAs have been known for decades for their function in genomic imprinting and chromosome inactivation respectively. Now they are being implicated in processes like cell proliferation and cell death, cell differentiation, nuclear transport etc. Their molecular mechanism of action mainly involves scaffolding activity in positioning necessary regulators of gene expression. The role of long noncoding RNAs have been characterized in greater details in most of the diseases, especially in cancer. Some reports are also there, describing possible involvement of lncRNAs in inflammatory diseases. However, there is not much information regarding the expression of IncRNAs in pancreatic cancer as well as chronic pancreatitis. The first study dates back to 1999 , where, there was an attempt to analyse the role of imprinting of H19 long noncoding RNA in human inflammatory pancreatic tissue and they found that H19 is imprinted in normal pancreas as well as in chronic pancreatitis (Micha et al., 1999). One study has compared the expression of both protein coding mRNAs as well as long noncoding RNAs in normal pancreas, PDAC and CP tissues and they could detect intergenic and intronic long noncoding RNAs in CP tissues along with known long noncoding RNAs (Tahira et al., 2011). Another group performed a similar long noncoding RNA profiling experiment in PDAC and CP tissue and reported that expression of HOTTIP and 6 other long noncoding RNAs were downregulated and that of 26 long noncoding RNAs were upregulated in $\mathrm{CP}$ as compared to PDAC (Wang et al., 2015). One of the mechanisms of long noncoding RNA mediated regulation of gene expression can be explained through 'ceRNA 
Table 1: Exploration of miRNA changes in pancreatic tissues and body fluids of CP patients with respect to healthy control individuals

\section{A. Comparison in pancreatic tissue}

Analysis of Global miRNA changes

\begin{tabular}{|c|c|c|c|}
\hline \multirow{2}{*}{$\begin{array}{l}\text { Study reference/ } \\
\text { Dataset reference }\end{array}$} & \multicolumn{2}{|l|}{ Top 10 deregulated miRNAs } & \multirow[b]{2}{*}{ Rema } \\
\hline & Upregulated & Downregated & \\
\hline $\begin{array}{l}\text { Bauer et al., 2012/ } \\
\text { GSE24279 }\end{array}$ & $\begin{array}{l}\text { miR-100, miR-361-3p, miR-181d, miR-514, } \\
\text { miR-181b, miR-532-3p, miR-181c, } \\
\text { miR-1206, miR-214,miR-486-5p }\end{array}$ & $\begin{array}{l}\text { miR-7, miR-217, miR-130b, miR-182, } \\
\text { miR-216a, miR-192, miR-215, } \\
\text { miR-216b, miR-194, miR-335 }\end{array}$ & $\begin{array}{l}\text { Comp } \\
\text { tissue } \\
\text { Norm }\end{array}$ \\
\hline $\begin{array}{l}\text { Wang et al., 2016/ } \\
\text { GSE24279 }\end{array}$ & $\begin{array}{l}\text { miR-155, miR-150, miR-181d, miR-181c, } \\
\text { miR-181a, miR-181b, miR-214, miR-100, } \\
\text { miR-31, miR-214* }\end{array}$ & $\begin{array}{l}\operatorname{miR}-548 b-3 p, \text { miR-429, } \\
\text { miR-216b, miR-216a, miR-130b, } \\
\text { miR-30a, miR-200b, miR-200c, } \\
\text { miR-375, miR-192 }\end{array}$ & Do \\
\hline $\begin{array}{l}\text { Bloomston et al., } \\
\text { 2007/E-TABM-664 }\end{array}$ & $\begin{array}{l}\text { miR-494, miR-100-1/2, miR-125b-2, miR- } \\
\text { 125b-1, miR-125a, miR-199a-1, miR-100, } \\
\text { miR-199a-2, miR-10b, miR-7-3 }\end{array}$ & miR-96, miR-497 & Do \\
\hline \multirow[t]{2}{*}{$\begin{array}{l}\text { Szafranska et al., } \\
2007\end{array}$} & $\begin{array}{l}\text { miR-150, miR-31, miR-145, miR-143, } \\
\text { miR-214, miR-125b, miR-100, miR-99a, } \\
\text { miR-487, miR-199a }\end{array}$ & $\begin{array}{l}\text { miR-148b, miR-96, miR-148a, } \\
\text { miR-130b, miR-29c, miR-95, } \\
\text { miR-182, miR-374, miR-216, miR-217 }\end{array}$ & Do \\
\hline & \multicolumn{3}{|c|}{ Analysis of Candidate miRNA Changes } \\
\hline \multicolumn{2}{|l|}{$\begin{array}{l}\text { Vychytilova- } \\
\text { Faltejskova } \text { et al., } 2015\end{array}$} & $\operatorname{miR}-217$ & Do \\
\hline \multicolumn{2}{|l|}{ Deng et al., 2014} & $\operatorname{miR}-217$ & Do \\
\hline Hu et al., 2015 & miR-34amiR-21 & & Do \\
\hline Natarajan et al., & $\operatorname{miR}-21$ & & Do \\
\hline
\end{tabular}

\section{B. Comparison in body fluid}

Analysis of Global miRNA Changes

Study reference Upregulated Downregated

Xin et al., $2017 \quad$ Early CP:miR-221, miR-130aLate CP: miR-320a, miR-320b, miR-320c, miR-320d

Comparison in serum of $\mathrm{CP}$ and Normal individuals

Debernardi et al., $\quad$ miR-30b, miR-30e, miR-143, miR-223

miR-4750, miR-3141, miR-4739,

Comparison in urine of 2015 miR-1207-5p, miR-1275, miR-149*, miR-1915, miR-665, miR-483-5p, miR-3663-3p, miR-204

Analysis of Candidate miRNA Changes

Liu et al., 2012 miR-155, miR-196a, miR-181a, miR-181b

Comparison in serum of $\mathrm{CP}$ and Normal individuals

Kong et al., $2011 \quad$ miR-21

Do

hypothesis' or competing endogenous RNA hypothesis, where the long noncoding RNA itself acts as the decoy of a specific miRNA to sponge it out, leaving the overexpression of the mRNAs which are target of that specific miRNA. Some recent studies have explored this phenomenon and interestingly, one of them has proposed a lncRNA-miRNA-mRNA ceRNA network involving long noncoding RNA Syntaxin-12, miR148a, miR-130b, SMAD5 or Interleukin 6 Signal Transducer (IL6ST), operating 
Table 2: Exploration of long noncoding RNA changes in pancreatic tissue of $\mathrm{CP}$ patients with respect to healthy control individuals

\section{A. Comparison in pancreatic tissue}

Analysis of Global lncRNA Changes

\begin{tabular}{|c|c|c|c|}
\hline \multirow{2}{*}{$\begin{array}{l}\text { Study reference/ } \\
\text { Dataset reference }\end{array}$} & \multicolumn{2}{|c|}{ Top 10 deregulated lncRNAs } & \multirow[b]{2}{*}{ Remarks } \\
\hline & Upregulated & Downregated & \\
\hline Wang et al., 2015 & $\begin{array}{l}\text { TMED11PTMED11PRP5-894D12.3H } \\
\text { MlincRNA767RP11-331F4.4RP4-809F } \\
\text { 18.1LINC00426RP11-753D20.4LOC } \\
\text { 389023AC011306.2 }\end{array}$ & $\begin{array}{l}\text { XLOC_006390HOTTIP-005RP11- } \\
\text { 567G11.1XLOC_012784LINC } \\
\text { 00460CTD-2547H18.1RP3- } \\
\text { 395M20.9 }\end{array}$ & $\begin{array}{l}\text { Comparison in } \\
\text { pancreatic tissue of } \\
\mathrm{CP} \text { and PDAC } \\
\text { patients }\end{array}$ \\
\hline \multicolumn{4}{|c|}{ Analysis of Candidate lncRNA Changes } \\
\hline Micha et.al., 1999 & H19 & & $\begin{array}{l}\text { Found to be imprinted in } \\
\text { both normal and } \mathrm{CP} \\
\text { pancreas }\end{array}$ \\
\hline Wang H et al., 2017 & STX12 & & $\begin{array}{l}\text { Comparison in pancreatic } \\
\text { tissue of } \mathrm{CP} \text { and PDAC } \\
\text { patients }\end{array}$ \\
\hline Li et al., 2016 & NUTF2P3-001 & & Do \\
\hline
\end{tabular}

during the PSC activation in chronic pancreatitis. They have also identified other putative ceRNA pairs regulating the activation of PSCs (Wang $\mathrm{H}$ et al., 2017). Another similar report elaborates the interaction involving long noncoding RNA, NUTF2P3-001, miR3923 and KRAS mRNA. Long noncoding RNA, NUTF2P3-001 is highly overexpressed in chronic pancreatitis tissue and shares the 3'-UTR binding site of miR-3923 with KRAS mRNA and their interaction involving ceRNA phenomenon describes a new mechanism of regulation of KRAS in CP and PDAC (Li et al., 2016). These deregulated IncRNAs are listed in Table 2.

\section{Future Perspectives}

Noncoding RNA mediated regulation of gene expression is one of the major post-transcriptional regulatory mechanism. They might also have multiple target genes and therefore, alteration of their levels might interfere with many pathways simultaneously. microRNAs and long noncoding RNAs are the two mostly studied noncoding RNAs and numerous studies have helped our understanding of the mechanism by which these noncoding RNAs could participate in disease pathogenesis. Furthermore, their identification as one of the major components of secretory exosomes has also initiated exploration to assess their potential to function as biomarkers for a specific disease. The importance of chronic pancreatitis disease lies in the fact that it is a progressive disorder of the pancreas leaving the patient in a morbid state with irreversible damage of the organ with minimal or no exocrine or endocrine activity. Furthermore, the disease has been considered as one of the major risk factors for pancreatic ductal adenocarcinoma. Hence several studies have been done to link two ends of the rope by exploring the role of ncRNAs in $\mathrm{CP}$, as discussed in this review and also summarized in Fig. 1. However, despite these efforts, gaps do exist in our knowledge, especially with respect to activation of pancreatic stellate cells and their mediation of subsequent events leading to the disease outcome. As newer noncoding RNAs, mostly long noncoding RNAs, are being discovered with the help of advanced technologies, it has become imperative to determine their exact functional role, elucidation of the biological pathways and signaling networks they interfere with, in greater details. It has been well documented that during the development and progression of diseases, ncRNAs can modulate expression of their target genes towards a specific fate and could themselves be regulated by specific disease conditions as well. In this context, another important aspect is to decode through appropriate experiments whether the disease specific 


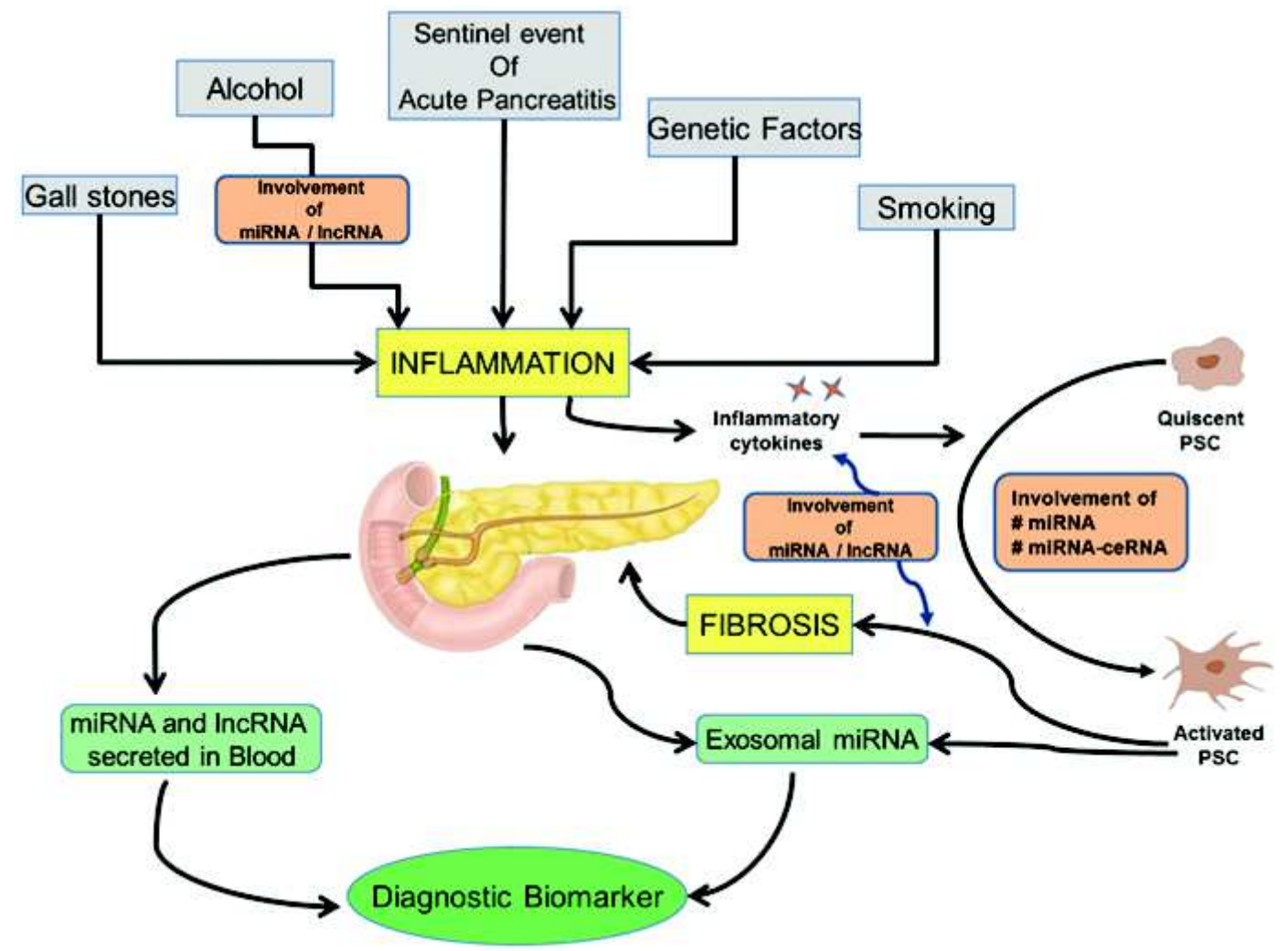

Fig. 1: Sequence of events during development of chronic pancreatitis and involvement of ncRNAs in the process: Illustrative diagram showing different causative agents initiating pancreatic tissue damage and inflammation of pancreas, leading to pancreatic stellate cell activation and fibrosis. Noncoding RNAs has been shown to modulate the entire process at multiple steps. Furthermore, secreted extracellular noncoding RNAs can also be explored for their biomarker potential. PSC stands for pancreatic stellate cells, miRNA: microRNA, IncRNA: long noncoding RNA and ceRNA: competing endogenous RNA

alterations in ncRNAs, described here, are the cause of disease or just a consequence of the disease pathophysiology. Moreover, additional efforts should be implemented to explore the biomarker potential of the noncoding RNAs in chronic pancreatitis as this area also has not been investigated fully.

\section{References}

Alexandar M and O'Connell R M (2015) Noncoding RNAs and chronic inflammation: Micro-managing the fire within Bioessays 37 1005-1015

Bai Y, Bai Y, Dong J, Li Q, Jin Y, Chen B and Zhou M (2016) Hedgehog Signaling in Pancreatic Fibrosis and Cancer Medicine 95 e2996

Balkwill F and Mantovani A (2001) Inflammation and cancer: back to Virchow? Lancet 357 539-545

\section{Acknowledgement}

None

\section{Funding}

The work has been performed receiving financial support from Department of Biotechnology, Government of India.

Barman K K, Premalatha G and Mohan V (2003) Tropical chronic pancreatitis Postgrad Med J79 606-615

Bauer A S, Keller A, Costello E, Greenhalf W, Bier M, Borries A, Beier M, Neoptolemos J, Büchler M, Werner J, Giese N and Hoheisel J D (2012) Diagnosis of Pancreatic Ductal Adenocarcinoma and Chronic Pancreatitis by Measurement of microRNAAbundance in Blood and Tissue PLoS One 7 e34151

BhanA, Soleimani M and Mandal S S (2017) Long Noncoding 
RNA and Cancer: A New Paradigm Cancer Research 15 3965-3981

Binkley C E, Zhang L, Greenson J K, Giordano T J, Kuick R, Misek D, Hanash S, Logsdon C D and Simeone D M (2004) The Molecular Basis of Pancreatic Fibrosis Common Stromal Gene Expression in Chronic Pancreatitis andPancreatic Adenocarcinoma Pancreas 29 254-263

Bloomston M, Frankel W L, Petrocca F, Volinia S, Alder H, Hagan J P, Liu C G, Bhatt D, Taccioli C and Croce C M (2007) MicroRNA Expression Patterns to Differentiate Pancreatic Adenocarcinoma From Normal Pancreas and Chronic Pancreatitis JAMA 297 1901-8

Carpenter S and Fitzgerald K A (2015) Transcription of Inflammatory Genes: Long Noncoding RNA and Beyond Journal of Interferon \& Cytokine Research 2 79-88

Charrier A, Chen R, Chen L, Kemper S, Hattori T, Takigawa M and Brigstock D R (2014) Connective tissue growth factor (CCN2) and microRNA-21 are components of a positive feedback loop in pancreatic stellate cells (PSC) during chronic pancreatitis and are exported in PSC-derived exosomes Journal of Cell Communication and Signaling $\mathbf{8}$ $147-56$

Cheng J, Guo J M, Xiao B X, Miao Y, Jiang Z, Zhou H and Li QN (2011) piRNA, the new non-coding RNA, is aberrantly expressed in human cancer cells Clinica Chimica Acta $\mathbf{4 1 2}$ 1621-1625

Clemens D L and Mahan J K (2010) Alcoholic pancreatitis: Lessons from the liver World Journal of Gastroenterology 16 1314-1320

Croce C M (2009) Causes and consequences of microRNA dysregulation in cancer Nature Review Genetics 10704 714

Debernardi S, Massat N J, Radon T P, Sangaralingam A, Banissi A, Ennis D P, Dowe T, Chelala C, Pereira S P, Kocher H M 6, Young B D, Bond-Smith G, Hutchins R and CrnogoracJurcevic T (2015) Noninvasive urinary miRNA biomarkers for early detection of pancreatic adenocarcinoma $A m J$ Cancer Res 5 3455-3466

Deng S, Zhu S, Wang B, Li X, Liu Y, Qin Q, Gong Q, Niu Y, Xiang C, Chen J, Yan J, Deng S, Yin T, Yang M, Wu H, Wang C and Zhao G (2014) Chronic pancreatitis and pancreatic cancer demonstrate active epithelial-mesenchymal transition profile, regulated by miR-217-SIRT1 pathway Cancer Letters 355 184-91

Derdak Z, Villegas K A, Harb R, Wu A M, Sousa A and Wands J $R$ (2013) Inhibition of p53 attenuates steatosis and liver injury in a mouse model of non-alcoholic fatty liver disease Journal of Hepatology 58 785-91
Dodge J A (1998) Pediatric and hereditary aspects of chronic pancreatitis Digestion 59 49-59

Esteller M (2011) Non-coding RNAs in human disease Nature Reviews Genetics 12 861-874

Forsmark C E (2013) Management of Chronic Pancreatitis Gastroenterology 144 1282-1291

Garg P K and Tandon R K (2004) Survey on chronic pancreatitis in the Asia-Pacific region Journal of Gastroenterology and Hepatology 19 998-1004

Hu L H, Ji J T and Li Z S (2015) Potential application of miRNAs as diagnostic and therapeutic tools in chronic pancreatitis Journal of Cell and Molecular Medicine 19 2049-57

Huang G, Li S, Yang N, Zou Y, Zheng D and Xiao T (2017) Recent progress in circular RNAs in human cancers Cancer Letters $4048 \mathrm{e} 18$

Kleff J, Whitcomb C D, Shimosegawa T, Esposito I, Lerch M M, Gress T, Mayerle J, Drewes A M, Rebours V, Akisik F, Muñoz J E D and Neoptolemos J P (2017) Chronic pancreatitis Nature Reviews: Disease primers volume $\mathbf{3}$ Article number 17060

Kong X, Du Y, Wang G, Gao J, Gong Y, Li L, Zhang Z, Zhu J, Jing Q, Qin Y and Li Z (2011) Detection of differentially expressed micro RNAs in serum of pancreatic ductal adenocarcinoma patients: miR-196a could be a potential marker for poor prognosis Digestive Diseases and Sciences $\mathbf{5 6}$ 602-9

Li X, Deng S J, Zhu S, Jin Y, Cui S P, Chen J Y, Xiang C, Li Q Y, He C, Zhao S F, Chen H Y, Niu Y, Liu Y, Deng S C, Wang C Y and Zhao G (2016) Hypoxia-induced lncRNANUTF2P3-001 contributes to tumorigenesis of pancreatic cancer by derepressing the miR-3923/KRAS pathway Oncotarget 7 6000-14

Liu J, Gao J, Du Y, Li Z, Ren Y, Gu J, Wang X, Gong Y, Wang W and Kong $X$ (2012) Combination of plasma microRNAs with serum CA19-9 for early detection of pancreatic cancer International Journal of Cancer 131 683-91

Marques-Rocha J L, Samblas M, Milagro F I, Bressan J, Martínez J A and Marti A (2015) Noncoding RNAs, cytokines, and inflammation-related diseases FASEB Journal 93 595-611

Masamune A, Nakano E, Hamada S, Takikawa T, Yoshida N and Shimosegawa T (2014) Alteration of the microRNA expression profile during the activation of pancreatic stellate cells Scandinavian Journal of Gastroenterology 49 323-31

Meng F, Glaser S S, Francis H, Yang F, Han Y, Stokes A, Staloch D, McCarra J, Liu J, Venter J, Zhao H, Liu X, Francis T, Swendsen S, Liu C G, Tsukamoto H and Alpini G (2012) 
Epigenetic regulation of miR-34a expression in alcoholic liver injury The American Journal of Pathology 181 804817

Micha A E, Hähnel S, Friess H, Büchler M W, Adler G and Gress T M (1999) Genomic Imprinting of IGF-II and H19 in Adult Human Pancreatic Tissues Digestion 60 477-483

Midha S, Khajuria R, Shastri S, Kabra M and Garg P K (2010) Idiopathic chronic pancreatitis in India: phenotypic characterisation and strong genetic susceptibility due to SPINK1 and CFTR gene mutations Gut 59 800-807

Mounzer R and Whitcomb D C (2013) Genetics of acute and chronic pancreatitis Curr Opin Gastroenterology 29544 551

Natarajan S K, Pachunka J M and Mott J L (2015) Role of microRNAs in Alcohol-Induced Multi-Organ Injury Biomolecules 53 309-38

Ptak-Belowska A, Targosz A and Brzozowski T (2012) MicroRNAs and Chronic Inflammation Contribution to Gastrointestinal Integrity Current Medicinal Chemistry 19 133-136

Rosendahl J, Bodeker H, Moosner J and Teich N (2007) Hereditary chronic pancreatitis Orphanet Journal of Rare Disease 21

Reddy D N and Ravi Kanth V (2014) Genetics of acute and chronic pancreatitis: An update World Journal of Gastrointestinal Pathophysiology 5 427-437

Schneider A and Whitcomb D C (2002) Hereditary pancreatitis: A model for inflammatory diseases of the pancreas Best Practice \& Research Clinical Gastroenterology 16347-63

Stevens T, Conwell D L and Zuccaro G (2004) Pathogenesis of Chronic Pancreatitis: An evidence based review of past theories and recent developments AmericanJournal for Gastroenterology 99 2256-2270

Strobel O, Buchler M W and Werner J (2009) Surgical therapy of chronic pancreatitis: Indications, techniques and results InternationalJournal of Surgery 7 305-312

Tahira A C, Kubrusly M S, Faria M F, Dazzani B, Fonseca R S, Maracaja-Coutinho V, Verjovski-Almeida S, Machado M C C and Reis E M (2011) Long noncoding intronic RNAs are differentially expressed in primary and metastatic pancreatic cancer Molecular Cancer 10141
Takikawa T, Masamune A, Yoshida N, Hamada S, Kogure T and Shimosegawa T (2017) Exosomes Derived From Pancreatic Stellate Cells MicroRNA Signature and Effects on Pancreatic Cancer Cells Pancreas 46 19-27

Vychytilova-Faltejskova P, Kiss I, Klusova S, Hlavsa J, Prochazka V, Kala Z, Mazanec J, Hausnerova J, Kren L, Hermanova M, Lenz J, Karasek P, Vyzula R and Slaby O (2015) MiR-21, miR-34a, miR-198 and miR-217 as diagnostic and prognostic biomarkers for chronic pancreatitis and pancreatic ductal adenocarcinoma Diagnostic Pathology 1038

Wang D, Xin L, Lin J H, Liao Z, Ji J T, Du T T, Jiang F, Li Z S and $\mathrm{Hu} \mathrm{L} \mathrm{H}$ (2017) Identifying miRNA-mRNA regulation network of chronic pancreatitis based on the significant functional expression Medicine (Baltimore) 96 e6668

Wang D, Zhu Z M, Tu Y L, Dou C Q, Xu Y, Tan X L, Han M M, Yang Z J, Jin X, Zhang B, Cai S and Liu Z W (2016) Identification of key miRNAs in pancreatitis using bioinformatics analysis of microarray data Molecular Medicine Reports 14 5451-5460

Wang H, Jiang Y, Lu M, Sun B, Qiao X, Xue D and Zhang W (2017) STX12 lncRNA/miR-148a/SMAD5 participate in the regulation of pancreatic stellate cell activation through a mechanism involving competing endogenous RNA Pancreatology 17 237-246

Wang Y, Zhihua Li Z, Zheng S, Zhou Y, Zhao L, Ye H, Zhao X, Gao W, Fu Z, Zhou Q, Liu Y and Chen R (2015) Expression profile of long non-coding RNAs in pancreatic cancer and their clinical significance as biomarkers Oncotarget 635684 35698

Xin L, Gao J, Wang D, Lin J H, Liao Z, Ji J T, Du T T, Jiang F, Hu LH and Li Z S (2017) Novel blood-based microRNA biomarker panel for early diagnosis of chronic pancreatitis Scientific Reports 74001

Zheng L, Xue J, Jaffee E M and Habtezion A (2013) Role of Immune Cells and Immune-Based Therapies in Pancreatitis and Pancreatic Ductal Adenocarcinoma Gastroenterology 144 1230-1240. 